\title{
Atrial Septostomy for Left Atrial Decompression During Extracorporeal Membrane Oxygenation by Inoue Balloon Catheter
}

\author{
Yen-Nien Lin, MD; Yin-Huei Chen, MD; Huang-Joe Wang, MD; \\ Jui-Sung Hung, PhD; Kuan-Cheng Chang, PhD; Ping-Han Lo, MD
}

\begin{abstract}
Background: Refractory pulmonary edema is an infrequent but serious complication in patients receiving venoarterial extracorporeal membrane oxygenation (VA-ECMO) for myocardial failure. Left atrial (LA) decompression in this setting is important. Although a few methods have been reported, the experience is mostly limited to children. We aimed to evaluate the feasibility of Inoue balloon catheter in percutaneous trans-septal LA decompression in adult cardiogenic patients.
\end{abstract}

\begin{abstract}
Methods and Results: We retrospectively analyzed 16 procedures of trans-septal LA decompression by Inoue balloon catheter in 15 VA-ECMO patients (aged 22-65 years, 6 men) with refractory pulmonary edema from May 2012 to December 2014. Mean left ventricular ejection fraction was $15 \%$. The cause of cardiogenic shock included 7 cases of ischemic heart disease, 1 of dilated cardiomyopathy, 5 of myocarditis, and 2 of fatal ventricular arrhythmia. The procedures were performed 4.3 days after ECMO. Inoue balloon size was $24-27 \mathrm{~mm}$. LA septostomy were successfully created in 14 patients. Procedure time on average was $36.8 \mathrm{~min}$ (range, $15-85 \mathrm{~min}$ ). There were no procedure-related complications. Radiography on the next day showed rapid resolution of pulmonary edema.
\end{abstract}

Conclusions: Trans-septal LA decompression by Inoue balloon catheter is a feasible alternative method for adult patients with refractory pulmonary edema under ECMO.

Key Words: Atrial septostomy; Extracorporeal membrane oxygenation; Left atrial decompression; Pulmonary edema; Trans-septal catheterization

V enoarterial extracorporeal membrane oxygenation (VA-ECMO) has been used in myocardial failure caused by acute myocardial infarction, myocarditis, decompensated cardiomyopathy, or intractable arrhythmia to provide immediate cardiac and respiratory support. ${ }^{1}$ However, in such persistent pump failure patients, the arterial cannula can place additional afterload on the left ventricle (LV) and lead to rising $\mathrm{LV}$ end-diastolic pressure, and left atrial (LA) pressure. Finally, refractory severe pulmonary edema might develop. In this setting, both the wall stress and the oxygen consumption of the LV increase, and recovery of LV function and weaning from ECMO will be delayed. ${ }^{2}$ Although not all patients on ECMO suffer from the described vicious circle, this complex medical condition does demand a solution to avoid additional complications.

LA decompression shows good results in minimizing LA/LV volume/pressure overload, chamber dilation, and resolving severe pulmonary edema. Early LA decompression was reported to enhance LV recovery. ${ }^{2} \mathrm{~A}$ few methods of achieving LA decompression have been reported, including balloon atrial septostomy, ${ }^{\mathbf{3 , 4}}$ combined blade and balloon atrial septostomy, ${ }^{5}$ trans-septal/transaortic LV sheath, ${ }^{6-8}$ transaortic pigtail LV drain, ${ }^{9}$ surgical LA/LV vent and trans-septal cannula incorporated into ECMO. ${ }^{10,11}$ However, the experience with these is mostly limited to children. Some methods are complex and destructive. Older individuals may have much thicker atrial septal walls, so whether the percutaneous balloon dilation method is feasible has been criticized. ${ }^{12}$ The Inoue balloon was specially designed for percutaneous transvenous mitral commissurotomy (PTMC), ${ }^{13}$ and the purpose of this article is to report our single-center experience with trans-septal LA decompression using the Inoue balloon catheter.

Received December 25, 2016; revised manuscript received April 4, 2017; accepted April 11, 2017; released online May 11, 2017 Time for primary review: 29 days

Division of Cardiovascular Medicine, Department of Medicine (Y.-N.L., H.-J.W., J.-S.H., K.-C.C., P.-H.L.), Division of Endocrinology and Metabolism, Department of Internal Medicine (Y.-H.C.), Cardiovascular Research Laboratory (Y.-N.L., H.-J.W., K.-C.C.), China Medical University Hospital, Taichung; Graduate Institute of Clinical Medical Science, China Medical University, Taichung (Y.-N.L., K.-C.C.), Taiwan

Mailing address: Ping-Han Lo, MD, Division of Cardiology, Department of Internal Medicine, China Medical University Hospital, No. 2 Yuh-Der Road, Taichung 40447, Taiwan. E-mail: lo.pinghan@gmail.com

ISSN-1346-9843 All rights are reserved to the Japanese Circulation Society. For permissions, please e-mail: cj@j-circ.or.jp 


\begin{tabular}{|c|c|c|c|c|c|c|c|c|}
\hline No. & $\begin{array}{c}\begin{array}{c}\text { Age } \\
\text { (years) }\end{array}\end{array}$ & Sex & $\begin{array}{l}\text { BW } \\
\text { (kg) }\end{array}$ & $\begin{array}{c}\text { Cause of } \\
\text { cardiogenic shock }\end{array}$ & $\begin{array}{l}\text { LVEF } \\
(\%)\end{array}$ & $\begin{array}{c}\text { Procedure } \\
\text { (days from ECMO) }\end{array}$ & $\begin{array}{c}\text { ECMO } \\
\text { days }\end{array}$ & $\begin{array}{l}\text { LVAD } \\
\text { use }\end{array}$ \\
\hline 1 & 54 & $M$ & 90 & ICMP & 14 & 7 & 15 & Y \\
\hline 2 & 58 & M & 60 & ICMP & 18 & 1 & 6 & $\mathrm{~N}$ \\
\hline 3 & 55 & M & 64 & ICMP & 11 & 11 & 22 & Y \\
\hline 4 & 37 & $M$ & 85 & ICMP & 7 & 2 & 9 & $\mathrm{~N}$ \\
\hline 5 & 40 & $M$ & 101 & DCMP & 10 & 7 & 35 & $Y$ \\
\hline 6 & 22 & M & 58 & VT & 21 & 7 & 13 & $N$ \\
\hline 7 & 49 & M & 82 & ICMP & 17 & 2 & 10 & $\mathrm{~N}$ \\
\hline 8 & 51 & $\mathrm{~F}$ & 55 & Myocarditis & 18 & 1 & 14 & $\mathrm{~N}$ \\
\hline 9 & 50 & $M$ & 73 & Myocarditis & 14 & 2 & 18 & $Y$ \\
\hline 10 & 65 & $\mathrm{~F}$ & 62 & ICMP & 21 & 6 & 12 & $\mathrm{~N}$ \\
\hline 11 & 40 & $\mathrm{~F}$ & 66 & VT & 15 & 2 & 5 & $\mathrm{~N}$ \\
\hline 12 & 58 & M & 70 & ICMP & 19 & 6 & 40 & $Y$ \\
\hline 13 & 62 & $\mathrm{~F}$ & 60 & Myocarditis & 21 & 5 & 28 & $\mathrm{~N}$ \\
\hline 14 & 51 & $\mathrm{~F}$ & 67 & Myocarditis & 10 & 2 & 19 & Y \\
\hline 15 & 32 & $\mathrm{~F}$ & 56 & Myocarditis & 10 & 3 & 17 & $\mathrm{~N}$ \\
\hline
\end{tabular}

DCMP, dilated cardiomyopathy; ECMO, extracorporeal membrane oxygenation; ICMP, ischemic cardiomyopathy; LVAD, left ventricular assisted device; VT, ventricular tachycardia.

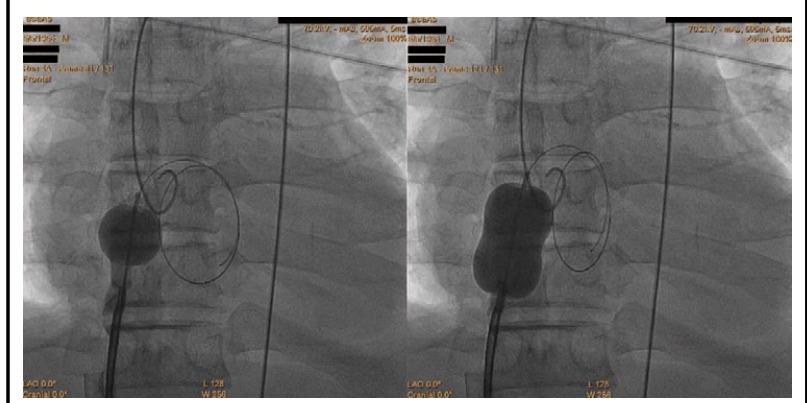

Figure 1. Inoue balloon inflation during septostomy. After the Inoue balloon catheter crossed the atrial septum, it was half inflated and pulled back until the distal balloon was leaning against the atrial septum, and then it was fully inflated.

\section{Methods}

Within May 2012 and December 2014, 124 patents suffered from cardiogenic shock and received VA-ECMO treatment at China Medical University Hospital. This retrospective study encompassed 15 ECMO patients who developed refractory severe pulmonary edema and underwent percutaneous trans-septal LA decompression. The demographic information is summarized in Table 1. Overall mean age was 48.3 years; 6 patients were men. Mean LV ejection fraction was $15 \%$. The procedures were performed 4.3 days after ECMO under fluoroscopy.

Institutional review board approval was obtained for analyzing patient demographics, catheterization reports, angiograms, echocardiograms, chest radiographs, ECMO flow data, and inpatient charts. Before the procedure, patients were informed about procedural risks, and therapeutic benefits. Written informed consent was obtained for all procedures.

\section{Interventional Techniques}

All 15 procedures were performed in the cardiac catheter- ization laboratory under fluoroscopic guidance. Vascular access was via the femoral vein (ipsilateral in 12 cases, contralateral in 3 cases). Trans-septal puncture was performed using a Brockenborough needle loaded in a Mullin sheath $\mathbf{1 4 , 1 5}$ under biplane fluoroscopic guidance (anteroposterior (AP) view and true lateral view). The position of the aortic valve was identified by its calcification ( 10 cases) or by the pigtail catheter ( 5 cases). The tip of the needle was directed posteriorly on the true lateral view and between 4 and 5 o'clock positions on AP view. After entering the LA, the Mullin sheath was advanced across the atrial septum and the needle was replaced with a stainless steel PTMC coiled guidewire. After the sheath was removed, the puncture wound, subcutaneous tissue, and the atrial septum were dilated by an 11F PTMC dilator. A 24-mm ( 8 cases) or 26-mm ( 6 cases) Inoue balloon catheter was then inserted across the atrial septum into the LA. After it was half inflated, it was pulled back until the distal balloon leaned against the atrial septum, and then it was fully inflated. Indentation of the atrial septum on the waist of the balloon was identified and then it disappeared after full inflation (Figure 1). Throughout the whole procedure, patients were under full anticoagulation for continuous ECMO without additional heparin. All patients underwent follow-up chest radiography and transthoracic echocardiography daily (Figure 2). The oxygenation index, defined as $\mathrm{FiO}_{2} \times$ mean airway pressure $\times 100 / \mathrm{PaO}_{2}$, before and after atrial septostomy was calculated for evaluation of respiratory status.

Procedural success was defined as successful creation of an artificial atrial septal defect (ASD) with full inflation of the Inoue balloon; a left-to-right shunt via the septal defect was determined by color-flow Doppler or contrast injection under fluoroscopy.

\section{Results}

Atrial septostomy was successfully performed in 14 patients with 16 procedures (Table 2). Patient 5 required a second procedure to create better inter-atrial communication because of delayed resolution of pulmonary edema. Patient 

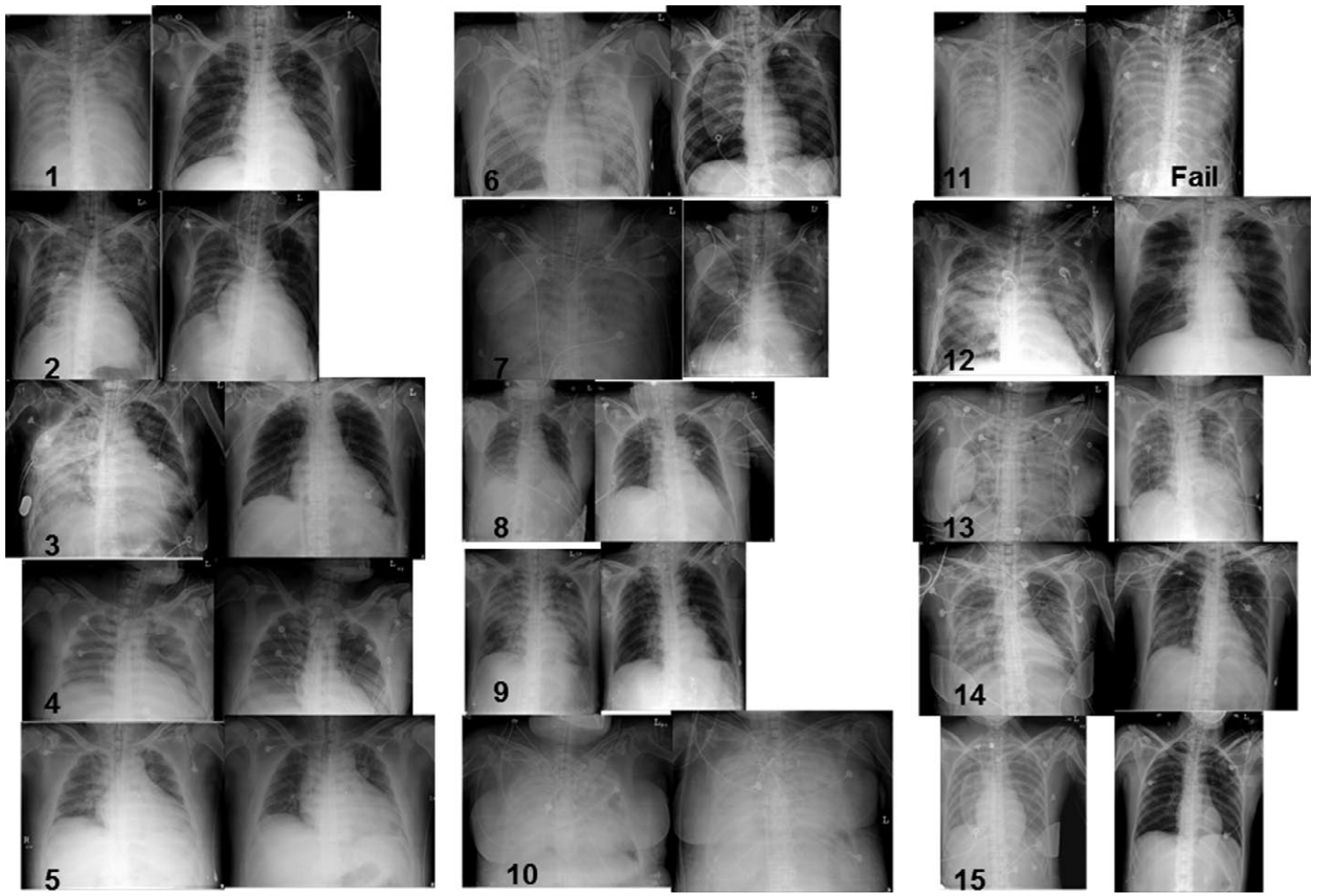

Figure 2. Supine anteroposterior chest radiography at 3 days after procedure. The refractory pulmonary edema improved after decompression of left atrium except in patients 10 and 11. Patient no.10 was complicated with pneumonia and adult respiratory distress syndrome. The transeptal puncture failed in patient no. 11 because of catheter kinking.

\begin{tabular}{|c|c|c|c|c|c|c|c|c|}
\hline No. & $\begin{array}{l}\text { BW } \\
\text { (kg) }\end{array}$ & $\begin{array}{c}\text { Access } \\
\text { (ipsi/contra) }\end{array}$ & $\begin{array}{l}\text { Procedure time } \\
\text { (min) }\end{array}$ & $\begin{array}{l}\text { Inoue balloon size } \\
(\mathrm{mm})\end{array}$ & $\begin{array}{l}\text { Death or cause } \\
\text { of death }\end{array}$ & HTX & $\begin{array}{l}\text { ASD } \\
\text { repair }\end{array}$ & $\begin{array}{c}\text { LVEF F/U } \\
(\%)\end{array}$ \\
\hline 10 & 90 & Contra & 25 & 24 & Sepsis & & & \\
\hline 2 & 60 & Contra & 45 & 24 & Sepsis & & & \\
\hline 13 & 64 & Ipsi & 20 & 26 & Sepsis & & & \\
\hline 4 & 85 & Ipsi & 60 & 26 & Sepsis & & & \\
\hline 5 & 101 & Ipsi & 20 & $26 / 27$ & Sepsis & & $Y$ & \\
\hline 7 & 58 & Ipsi & 30 & 24 & Sepsis & & & \\
\hline 11 & 82 & Ipsi & Fail & Fail & IBD & & & \\
\hline 1 & 55 & Ipsi & 30 & 26 & $\mathrm{~N}$ & Y & $Y$ & \\
\hline 14 & 73 & Ipsi & 15 & 26 & $\mathrm{~N}$ & Y & $Y$ & \\
\hline 12 & 62 & Ipsi & 20 & 26 & $\mathrm{~N}$ & Y & $Y$ & \\
\hline 3 & 66 & Ipsi & 60 & 26 & $\mathrm{~N}$ & Y & $Y$ & \\
\hline 6 & 70 & Contra & 85 & 24 & $\mathrm{~N}$ & $\mathrm{~N}$ & $\mathrm{~N}$ & 55 \\
\hline 8 & 60 & Ipsi & 40 & 24 & $\mathrm{~N}$ & $\mathrm{~N}$ & $Y$ & 71 \\
\hline 9 & 67 & Ipsi & 30 & 26 & $\mathrm{~N}$ & $\mathrm{~N}$ & $Y$ & 60 \\
\hline 15 & 56 & Ipsi & 30 & 24 & $\mathrm{~N}$ & $\mathrm{~N}$ & $\mathrm{~N}$ & 42 \\
\hline
\end{tabular}

ASD, atrial septal defect; IBD, ischemic bowel disease; Contra, contralateral; HTx, heart transplantation; Ipsi, ipsilateral.

6 had the longest procedure time $(85 \mathrm{~min})$ because of difficulty in localizing the ideal trans-septal puncture point. Trans-septal puncture failed in patient 11 because of kinking of the device and venous cannula. The average procedure time was $36.8 \mathrm{~min}$ (range $15-85 \mathrm{~min}$ ).
All procedures were performed in critically ill patients. For the ECMO support system, vascular access was sometimes limited. The procedures via contralateral femoral vein showed $100 \%$ success rate and median procedure time of $45 \mathrm{~min}$ (ipsilateral femoral vein: success rate $91.7 \%$ and 


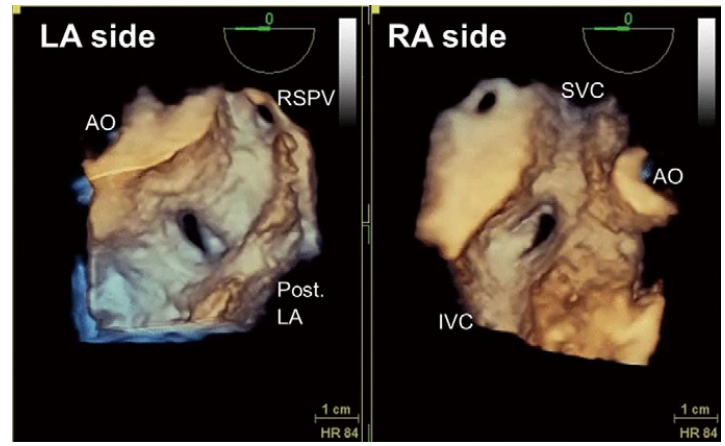

Figure 3. Transesophageal echocardiography 3D reconstruction for evaluation of artificial atrial septal defect (ASD). Five patients underwent transesophageal echocardiography during follow-up and 3D reconstruction was performed in 1 case. The artificial ASD was coin slot-shaped, with an area of $0.78 \mathrm{~cm}^{2}$. The average ASD diameter in the 5 cases was $7.5 \mathrm{~mm}$. AO, aorta; IVC, inferior vena cava; LA, left atrium; Post, posterior; RA, right atrium; RSPV, right superior pulmonary vein; SVC, superior vena cava.

median procedure time $30 \mathrm{~min}$ ). In the first 2 procedures, we performed trans-septal puncture with a pigtail catheter placed in the non-coronary aortic sinus for guidance. In the later procedures, most trans-sepal punctures, except in 3 patients who were young, were completed using aortic calcification as a landmark to save additional arterial puncture. To create an adequate ASD for left-to-right shunt, in patients with a body weight $>60 \mathrm{~kg}$, we chose the 26-mm Inoue balloon, except in 2 cases: the patient was short in height in 1 case and there was a lack of 26-mm balloons on procedure day in the other. In such cases, despite using a smaller balloon, the pulmonary edema resolved rapidly. However, in 1 case of a patient weighing $101 \mathrm{~kg}$, a second procedure with a $27-\mathrm{mm}$ Inoue balloon was required to make a larger ASD.

\section{Follow-up}

All patients were followed up in hospital for outcomes after the procedure (Table 2). There were no procedurerelated complications except for artificial ASD. Follow-up radiography in 3 days showed significant resolution of pulmonary edema (Figure 2). The oxygenation index also dropped from $9.9 \pm 5.94$ to $4.6 \pm 2.99(\mathrm{P}<0.001$ by paired t-test). Six patients were successfully weaned from mechanical ventilator and received a LV assist device (LVAD) in place of ECMO. Three of the other patients regained LV function and had ECMO successfully removed. Among the 6 patients who received LVAD, 4 underwent heart transplantation and recovered normal function. One regained LV function and was finally independent of the LVAD. In total, one of the patients who received LVAD and another 5 patients were complicated with sepsis and died. Patient 11 with an unsuccessful procedure died of ischemic bowel disease.

As to follow-up of the artificial ASD (Figure 3), 7 patients underwent ASD repair: 6 underwent the repair during LVAD implantation to avoid right-to-left shunt and the 7th patient underwent ASD repair under concerns of future complication of significant left-to-right shunt. However, among the surviving patients, 2 declined ASD repair.
Echocardiography at 1 year after hospitalization still showed left-to-right shunt but neither right heart enlargement nor pulmonary hypertension. They were free of symptoms.

\section{Discussion}

This study is the first reported adult series of percutaneous trans-septal LA decompression with the Inoue balloon to date. Despite the small number of patients, successful creation an efficient left-to-right shunt without complication in 14 of 15 patients demonstrated the feasibility and safety of the Inoue balloon in LA decompression. The whole procedure was performed under fluoroscopy in the catheterization laboratory with low total procedure times.

Although several methods had been reported in the previous literature, the experience is mostly limited to children. ${ }^{16}$ Compared with surgical methods or strategies utilizing the cannula connected to the ECMO system, balloon atrial septostomy is simpler and minimally invasive. However, the feasibility of balloon dilation method in older individuals, because they have a much thicker atrial septal wall. ${ }^{12}$ Thus a larger size balloon with good radial force might overcome this problem. In 1982 the Inoue balloon was specially designed for PTMC, ${ }^{13}$ because persistent ASD was a well-known complication of that procedure. ${ }^{17}$ With its special design, the Inoue balloon has been used in tricuspid stenosis, ${ }^{18}$ aortic stenosis, ${ }^{19}$ and, similar to our series, atrial septostomy ${ }^{20}$ for severe pulmonary hypertension and achieved good results.

The size of the LA drain or ASD determines satisfactory decompression. Aiyagari et $\mathrm{al}^{\mathbf{1 0}}$ reported that a patient with the largest sheath placed had the most rapid resolution of LA hypertension; 2 unsuccessful procedures had been performed with smaller LA cannulas. The Inoue balloon was used for its unique design of a self-seating mechanism. Upsizing can be done with the same balloon or by using a graded balloon dilatation technique. This advantage can further contribute to higher procedural success rates. In our reported series, the 24- or 26-mm Inoue balloon achieved adequate LA decompression in most patients. Based on this method, we do think other large balloons, in addition to the Inoue balloon, could achieve a similar result. However, we did not directly compare the efficacy between such balloons.

The benefit of LA decompression is mainly based on 2 main mechanisms. The first, reduction of LA pressure, promotes early lung recovery and reduces the duration under sedation and ventilation. In our study, because all procedures were performed in critically ill patients, we were not able to perform routine hemodynamic measurement. Three of the study patients had LA pressure measured by trans-septal puncture needle and the average LA pressure was $29 \mathrm{mmHg}$ before the procedure and reduced to $13 \mathrm{mmHg}$ immediately after atrial septostomy. This suggested an adequate ASD formed a left-to-right shunt that diverted blood flow from LA back to the ECMO and finally removed the accumulated fluid from the lung parenchyma. The second, ameliorated LV wall stress, improves subendocardial perfusion, decreases myocardial oxygen consumption, and subsequently helps LV functional recovery. So far, there is no consensus with regard to the timing of LA decompression during ECMO. However, studies generally conclude earlier LA decompression may be beneficial to minimize risks related to prolonged ECMO. ${ }^{2}$ 
In our study, we did not try to show that the Inoue balloon was superior to other existing LA decompression procedures because such head-to-head comparisons in the study patients were impossible. Based on our experience, trans-septal decompression by Inoue balloon was a simpler, minimally invasive procedure, had a short procedure time, a high procedure success rate, and the specially designed balloon was suitable for adults. We think this method may be an alternative to existing LA decompression for patients with refractory pulmonary edema under ECMO. However, despite the success of the procedure, we do not think LA decompression affected the clinical outcomes. In our study, the outcomes of the patients depended largely on the primary etiology of cardiogenic shock.

\section{Study Limitations}

First, this study was limited by its retrospective design and the small number of patients of study interest (those suffering from refractory pulmonary edema under ECMO support). We will continuously enroll patients and do further investigation. Second, because of the heterogeneous, complex, and critical medical conditions, we were unable to perform detailed hemodynamic measurements and close follow-up in each patient. Therefore, we cannot offer a strong underlying hemodynamic mechanism or molecular evidence. Third, although all patients were on ECMO for myocardial failure, this was an extremely heterogeneous group of patients with respect to age, body size, concomitant diseases, and prior cardiac disease as well as surgery. The validity of the statistical results may be affected.

\section{Conclusions}

In patients with persistent pulmonary edema after ECMO, effective LA decompression can be achieved using the Inoue balloon catheter and atrial septostomy.

\section{Acknowledgments}

This study was supported in part by the Taiwan Ministry of Health and Welfare Clinical Trial and Research Center of Excellence (MOHW105-TDU-B-212-133019).

\section{Conflict of interest}

No potential conflicts of interest declared.

\section{References}

1. Sayer GT, Baker JN, Parks KA. Heart rescue: The role of mechanical circulatory support in the management of severe refractory cardiogenic shock. Curr Opin Crit Care 2012; 18: 409-416.

2. Kotani Y, Chetan D, Rodrigues W, Sivarajan VB, Gruenwald C, Guerguerian AM, et al. Left atrial decompression during venoarterial extracorporeal membrane oxygenation for left ventricular failure in children: Current strategy and clinical outcomes. Artif Organs 2013; 37: 29-36.

3. Koenig PR, Ralston MA, Kimball TR, Meyer RA, Daniels SR, Schwartz DC. Balloon atrial septostomy for left ventricular decompression in patients receiving extracorporeal membrane oxygenation for myocardial failure. J Pediatr 1993; 122: S95S99.

4. Johnston TA, Jaggers J, McGovern JJ, O’Laughlin MP. Bedside transseptal balloon dilation atrial septostomy for decompression of the left heart during extracorporeal membrane oxygenation. Catheter Cardiovasc Interv 1999; 46: 197-199.

5. Seib PM, Faulkner SC, Erickson CC, Van Devanter SH, Harrell JE, Fasules JW, et al. Blade and balloon atrial septostomy for left heart decompression in patients with severe ventricular dysfunction on extracorporeal membrane oxygenation. Catheter Cardiovasc Interv 1999; 46: 179-186.

6. Cheung MM, Goldman AP, Shekerdemian LS, Brown KL, Cohen GA, Redington AN. Percutaneous left ventricular "vent" insertion for left heart decompression during extracorporeal membrane oxygenation. Pediatr Crit Care Med 2003; 4: 447449.

7. Fumagalli R, Bombino M, Borelli M, Rossi F, Colombo V, Osculati $\mathrm{G}$, et al. Percutaneous bridge to heart transplantation by venoarterial ECMO and transaortic left ventricular venting. Int J Artif Organs 2004; 27: 410-413.

8. Hong TH, Byun JH, Lee HM, Kim YH, Kang GH, Oh JH, et al. Initial experience of transaortic catheter venting in patients with venoarterial extracorporeal membrane oxygenation for cardiogenic shock. ASAIO J 2016; 62: 117-122.

9. Barbone A, Malvindi PG, Ferrara P, Tarelli G. Left ventricle unloading by percutaneous pigtail during extracorporeal membrane oxygenation. Interact Cardiovasc Thorac Surg 2011; 13: $293-295$.

10. Aiyagari RM, Rocchini AP, Remenapp RT, Graziano JN. Decompression of the left atrium during extracorporeal membrane oxygenation using a transseptal cannula incorporated into the circuit. Crit Care Med 2006; 34: 2603-2606.

11. Alkhouli M, Narins CR, Lehoux J, Knight PA, Waits B, Ling FS. Percutaneous Decompression of the left ventricle in cardiogenic shock patients on venoarterial extracorporeal membrane oxygenation. J Card Surg 2016; 31: 177-182.

12. Park SC, Neches WH, Mullins CE, Girod DA, Olley PM, Falkowski G, et al. Blade atrial septostomy: Collaborative study. Circulation 1982; 66: 258-266.

13. Inoue $\mathrm{K}$, Owaki T, Nakamura T, Kitamura F, Miyamoto N. Clinical application of transvenous mitral commissurotomy by a new balloon catheter. J Thorac Cardiovasc Surg 1984; 87: 394402.

14. Hung JS. Atrial septal puncture technique in percutaneous transvenous mitral commissurotomy: Mitral valvuloplasty using the Inoue balloon catheter technique. Cathet Cardiovasc Diagn 1992; 26: $275-284$.

15. Hung JS, Lau KW. Trans-septal access in Inoue-balloon mitral valvuloplasty. Indian Heart $J$ 2006; 58: $463-465$.

16. Eastaugh LJ, Thiagarajan RR, Darst JR, McElhinney DB, Lock JE, Marshall AC. Percutaneous left atrial decompression in patients supported with extracorporeal membrane oxygenation for cardiac disease. Pediatr Crit Care Med 2015; 16: 59-65.

17. Korkmaz S, Demirkan B, Guray Y, Yilmaz MB, Sasmaz H. Long-term follow-up of iatrogenic atrial septal defect: After percutaneous mitral balloon valvuloplasty. Tex Heart Inst $J$ 2011; 38: $523-527$.

18. Yokoyama Y, Abe M, Fujieda H, Abe Y. A rare case: Bioprothetic tricuspid valvuloplasty dramatically improved hepatic encephalopathy. Gen Thorac Cardiovasc Surg 2016; 64: 618-620.

19. Kato H, Kubota S, Goto T, Haba T, Yamamoto M. Externalization of a stiff guide wire via the radial artery: A new technique to facilitate advancement of an Inoue balloon across the aortic valve in patients with aortic stenosis undergoing antegrade balloon aortic valvuloplasty. Cardiovasc Interv Ther 2016; 31: 140-146.

20. Kapoor A, Khanna R, Batra A, Kumar S. Inoue balloon atrial septostomy in severe persistent pulmonary hypertention following surgical ASD closure. J Cardiol Cases 2012; 6: e1-e3. 